\title{
Pain scores of odontogenic orofacial lesions in a tertiary Hospital in sub Saharan West Africa.
}

\author{
Kolude Bamidele ${ }^{1}$ Gbadebo Shakeerah. ${ }^{2}$ \\ ${ }^{I}$ Department of Oral Pathology/Oral Medicine \\ ${ }^{2}$ Department of Restorative Dentistry ; University College Hospital (UCH)/College of medicine University of \\ Ibadan (UI), Ibadan, Nigeria.
}

\begin{abstract}
Aim \& objective To determine the correlation of three main pain measuring scales used as diagnostic aid to orofacial lesion of odontogenic origin.

Methodology A six month cross sectional survey utilizing interviewer administered questionnaire targeted at attendees of Dental outpatient clinic of University College Hospital, Nigeria (UCH), a tertiary hospital.

Results Mean age for odontogenic pain was $35.68 \pm 16.44$; male to female ratio was 1: 1.24.

Pain was more severe but statistically insignificant among females $(p=0.85)$, however, there was significant difference in the mean pain intensity between diagnostic categories of odontogenic lesion $(p=0.00)$. The commonest odontogenic lesion category was caries related (54.3\% of all orofacial and 58.9\% of odontogenic lesions respectively).

There was positive correlation between the three pain measuring scales (Visual Analogue Scale (VAS),Number of verbs chosen(NWC) \&MPQ) for odontogenic lesions ( $r=0.587 ; r=0.838 \& r=0.599$ respectively). However the three diagnostic scales failed to distinguish between specific odontogenic lesions when the mean pain scores were very close.
\end{abstract}

Conclusion Pain measuring scales should only be used as aid to diagnosis and not for definitive diagnosis.

Key words: Pain scales, odontogenic lesions, orofacial pain.

\section{Introduction}

Orofacial pain (OFP) is a common symptom that is experienced by a quarter of adult population ${ }^{1,2}$ and toothache is the most common symptom ${ }^{3,4}$. Odontogenic type of orofacial pain arise as a result of disorders of the tooth forming structures while the non odontogenic types arise from other structures that are not involved in tooth formation.

Previous studies on odontogenic orofacial pain included that of Van Buren $1979^{5}$ who evaluated the use of McGill Pain Questionnaire (MPQ) ${ }^{6}$ for dental pain assessment and by utilising the anxiety, affective, evaluative and present pain intensity (PPI) components of MPQ, concluded that MPQ was effective in describing dental pain syndrome since different groups of dental patients from various centres reported similar scores of MPQ subscale.

Seymour $1985^{7}$ used MPQ, visual analogue scale (VAS) and verbal description to compare pain severity between patients with pulpitis and those with pericoronitis and concluded that pain scale was able to distinguish pain of pulpitis from that of pericoronitis.

In an experimental study on age, gender and dental pain perception by Fagade et al $2004^{8}$, that utilised calibrated vitality pulp tester as measuring instrument, it was observed that middle aged and older patients presented significantly lower pain threshold than the adolescent and young patients, this observation was ascribed to the enhanced pain of anxiety due to previous dental treatment experience. While the works of MacMillian et al and Fagade et al ${ }^{4,8}$, observed no sex differences, Seymour 1985 \& Vickers $1998^{7,9}$ observed significant sex differences in pain intensity.

To the best of the authors' knowledge, no other previous work on prevalence and comparative measurement of orofacial pain perception has been done in sub Saharan west Africa, despite the frequent presentation of odontogenic orofacial pain in dental clinics, thus there was the need to see the demographic pattern of presentation of different types of odontogenic orofacial pain, as well as the severity of the different types of orofacial pain, and to determine whether the measurement of severity of pain may aid diagnosis.

The objective of this study was to make a comprehensive assessment of pain severity by utilising pain rating scales of MPQ, VAS and Number of Words Chosen (NWC) in patients presenting various types of odontogenic lesions and to further assess inter instrumental correlation of pain scores with the sole aim of utilising pain rating scales as diagnostic aid of odontogenic lesions. 


\section{Materials And Methods}

This study is a descriptive cross sectional survey of patients that presented at the Oral Diagnosis (OD) clinic, dental centre, University College Hospital Nigeria over a six month period from April to September 2011. The study centre is a tertiary hospital located in Ibadan, the largest city in West Africa. It is an urban area with a population of over 5 million. The hospital services are both free (through health Insurance scheme) and paid visits. OD clinic receive first time patients and those presenting after six months of previous dental visit.

House Officers and resident doctors were trained to interpret and administer the structured questionnaire at OD clinic. Data was collected through interviewer administered, structured questionnaire containing the following items: (1) Patient's data of age, sex, marital status, educational status and occupation. (2) Pain characteristics such as aetiology, family history, location, duration, trigger aggravating or relieving factors, clinical diagnosis and treatment modalities. A copy each of the following pain rating scales was also incorporated in the structured questionnaire: (3) Visual Analogue Scale (VAS) in which patient presenting with pain drew the line that best describe the present pain being experienced on a linear scale such that $0 \mathrm{~cm}$ represents no pain and $10 \mathrm{~cm}$ represents the worst pain imaginable.

MPQ, consist primarily of three major classes of word descriptors- sensory, affective and evaluative that are used by patient to qualify pain experience. It also contains both space and time pain descriptors and pain intensity scale. The MPQ has 78 words describing pain, categorised into 20 groups that represent the four major dimensions of pain quality, sensory, affective, evaluative and miscellaneous pain descriptions ${ }^{6}$.

NWC assess pain severity by counting the number of words used to describe such pain experience. Participants could choose words from the verbal descriptors of pain in MPQ or utilise other words to express pain perception. Vickers et al (2000) ${ }^{10}$ observed that NWC count was proportional to pain severity.

All data was analysed using the Window based Statistical Package for Social Sciences (SPSS) software version 11.0. Qualitative variables of gender, educational status and occupation were expressed as proportion and compared using chi square statistics while quantitative variables of age and pain scores were summarised as means, standard deviation and confidence interval while strength of association between variables were determined using student's t test, Pearson's correlation and/or analysis of Variance (ANOVA) Test.

\section{Results}

A total of 426 patients were seen in Oral diagnosis/Medicine clinic over the 6 month study period, spanning April to September 2011. Three hundred and nineteen patients presented with orofacial pain while 107 patients had no orofacial pain, giving orofacial prevalence rate of $74.88 \%$ among patients presenting at the Dental clinic U.C.H. Three hundred respondents with orofacial pain had properly filled questionnaires while 85 respondents without orofacial pain had properly documented questionnaire giving a response rate of $90.4 \%$ A total of 300 respondents filled the questionnaires properly and were included in the final analysis. There were 133 males and 167 females giving a male-to-female ratio of $1: 1.3$. Two hundred and seventy seven $(92.3 \%)$ presented with odontogenic lesions (dental origin) consisting of 122 males and 155 females, while 23 (7.7\%) presented with non-odontogenic lesions.

There was no statistically significant difference in the distributions of $\operatorname{sex}\left(X^{2}=0.15 ; p=0.70\right)$ age group $\left(X^{2}=\right.$ $0.36 ; \mathrm{p}=0.91)$ and marital status $\left(\mathrm{X}^{2}-0.02, \mathrm{p}=0.89\right)$ among orofacial pain patients when compared with those without.

\subsection{Socio-demographic pattern of the participants (Table 1)}

The age range of patients presenting with odontogenic lesions was 13-100 years with mean \pm SD of $35.68 \pm 16.44$ years. The peak age of occurrence of odontogenic lesions was in the 3rd decade $(35.1 \%)$ and the male to female ratio was 1: 1.24. There were no significant differences in the gender distribution of respondents according to age group $\left(p=0.822 ; X^{2}=2.89\right)$ and educational status $\left(X^{2}=6.43 ; p=0.093\right)$, there was however a significant difference in distribution of marital status according to gender $\left(P=.036 ; \mathrm{X}^{2}=10.29\right)$.

\subsection{Categories of Odontogenic lesions with orofacial Pain (Fig. 1)}

Caries related lesions - A total of 166 cases accounting for 59.9\% of all odontogenic were in this category, and comprised of apical periodontitis which was the most important cause of odontogenic pain accounting for (110) $66.3 \%$ of cases, followed by pulpitis occuring in $40(24.1 \%)$ of cases, this was followed by pain as a result of dentoalveolar abscess and submandibular cellulitis.

3.3 Gingival and Periodontal lesions - These lesions were recorded in 75 participants and accounted for $27.1 \%$ of all cases. The most frequent lesion causing periodontal pain was chronic periodontitis in 32 participants (42.7\%), followed by pericoronitis in 29 participants (37.8\%) and periodontal abscess in 5 participants $(6.8 \%)$. There were 3 cases of pain from retained root (4\%), while pain due to gingivitis, retained deciduous and 
Pain scores of odontogenic orofacial lesions in a tertiary Hospital in sub Saharan West Africa.

supplemental tooth occurred in 2 patients each, accounting for $2.7 \%$ of periodontal problem respectively. Only one patient $(1.4 \%)$ presented with gingival abscess.

3.4 Traumatic dental injury - In this group, 16 cases accounting for 5.8\% of all the cases were recorded. The predominant type of injury in the group was complicated crown fracture which accounted for 12 cases $(75 \%)$. This was followed by 2 cases of uncomplicated crown fracture involving only enamel (12.5\%) and a case each of complicated crown root fracture and subluxation injury.

3.5 Tooth surface loss - Twelve cases were recorded accounting for $4.3 \%$ of total. The predominant type of tooth surface loss was cervical abrasion occurring in 9 patients $(75 \%)$. This was followed by 2 cases of attrition $(16.7 \%)$ and one case of erosion (8.3\%) all of which resulted in dentinal sensitivity.

Faulty Restorations - The cause of pain from failed restoration was failed amalgam filling secondary to fractured restoration occurring in 8 patients accounting for $2.9 \%$ of all cases.

3.6 Assessment of pain severity and correlations using measuring scales.

3.6.1 The visual analogue scale - Using the scale of 1 to 10 for pain severity, the overall mean score for all odontogenic lesions was 5.69. According to disease categories, the patients that presented with caries related lesions had the highest mean score of $6.01 \pm 2.05$ while patients that presented with tooth surface loss had the least mean score of $3.33 \pm 1.15$.

3.6.2 Verbal Description of Pain Severity (NWC):The overall mean number of verbs used to describe the pain from odontogenic lesions was $2.34 \pm 0.84$; pain from caries related lesion types was described with highest number of verbs with mean score of $2.46 \pm 0.86$ while failed/incomplete restoration was described with the least no of verbs.

3.6.3 MPQ scores of Pain - According to McGill Pain Questionnaire, the overall mean score of pain for odontogenic lesions (PRI T) was 26.66 \pm 12.10 ; the category with the most severe pain was the traumatic dental injury group with mean score of 30 . This was followed in severity by caries related group with mean score of $28.66 \pm 12.10$. The least severe pain according to MPQ scores was observed in the group with tooth surface loss $(17.83 \pm 7.00)$.

3.6.4 Analysis of Variance (ANOVA) Comparison of mean Pain Scores of the diagnostic Categories of Odontogenic lesions (Table II) - One way analysis of variance comparison of mean pain scores between the three pain measuring Scales (VAS, MPQ \&NWC) according to diagnosis categories showed significant mean differences between the various diagnostic categories of odontogenic lesions. (NWC F=3.819, $\mathrm{p}=0.005$, VAS F $=7.521, \mathrm{p}=0.000, \mathrm{MPQ} F=4.867, \mathrm{p}=0.001$ )

3.6.5 Relationship between the scores of VAS and NWC (fig 2) - There was a statistically significant positive correlation between the scores of VAS and number of verbs used to describe odontogenic type of orofacial pain $(\mathrm{r}=0.587, \mathrm{t}=-27.85 ; \mathrm{C} . \mathrm{I}=-3.58$ to $-3.12 ; \mathrm{P}=0.00)$.

3.6.6 Relationship between the scores of VAS and MPQ (Fig 3) - There was also a statistically significant positive correlation between visual analogue pain score and MPQ for Odontogenic pain. $(\mathrm{r}=0.838, \mathrm{p}=0.00, \mathrm{t}$ $=33.287$; $\mathrm{C} . \mathrm{I}=19.95$ to 22.45 ).

3.6.7 Relationship between the scores of NWC and MPQ (Fig 4) - Again, a statistically significant positive correlation was observed between MPQ and number of verbs chosen for odontogenic pain. $(r=0.599, p=0.00$, $\mathrm{t}=34.13 ;$ C. $\mathrm{I}=22.91$ to 25.72 ).

ANOVA comparison of Mean Scores of Present Pain Intensity (PPI) and total MPQ scores (PRI T) between Disease Categories and Gender showed a significant difference in the mean scores of PPI and PRIT scores according to disease categories $(\mathrm{F}$ ratio $\& \mathrm{P}$ value of $\mathrm{PPI}=5.96 \& 0.00 ; \mathrm{F}$ ratio $\&$ P value of $\mathrm{PRIT}=4.85$ $\& 0.00$ ) but no significant difference in the mean scores of PPI, PRIT according to gender (F ratio \& $\mathrm{P}$ value of $\mathrm{PPI}=0.038 \& 0.85 ; \mathrm{F}$ ratio $\& \mathrm{P}$ value of PRIT $=1.98 \& 0.16$ ). This suggests that the mean present pain intensity (PPI) and the mean total MPQ pain scores were influenced by the disease categories but not by gender.

3.7 Utility of Pain rating Scales as Diagnostic aid for Odontogenic Orofacial lesion - None of the three pain measuring scales was able to distinguish between the 2 most common causes of odontogenic pain (pulpitis and apical periodontitis). $(\mathrm{F}=1.068, \mathrm{p}=0.303)$, neither was any of the scales able to distinguish between chronic 
Pain scores of odontogenic orofacial lesions in a tertiary Hospital in sub Saharan West Africa.

periodontitis and pericoronitis $(\mathrm{F}=0.856, \mathrm{p}=0.430)$. The three pain measurement scales were also unable to distinguish between pulpitis and pericoronitis. $(\mathrm{F}=1.565, \mathrm{p}=0.213)$.

\section{Discussion}

The commonest type of orofacial pain in the present study was of odontogenic origin, The finding that the overwhelming majority of cases of orofacial pain were of odontogenic origin in this study compared to the non odontogenic type may have to do with the study centre as majority of non odontogenic orofacial pain often present first to the physician for treatment rather than dental surgeons at UCH Ibadan.

The present study showed that more females presented at the Dental clinic, U.C.H Ibadan, compared with males, this may explain the higher proportion of females presenting with orofacial pain. This is in keeping with several studies that had documented more female presentation with orofacial pain ${ }^{6,11}$. Two studies in Australian by Gerschman et $\mathrm{al}^{12} \&$ Bush et $\mathrm{al}^{13}$ had previously presented an extreme gender difference in the female to male ratio of 3:1 for orofacial pain which was attributed to the lower pain threshold and better health motivation of females that resulted in a higher prevalence of females who 'actively' seek treatment for health complaints generally ${ }^{7}$.

There was however no significant difference in the mean pain score of females compared to males in keeping with findings of Vickers et $\mathrm{al}^{9}$. \& Van Buren et $\mathrm{al}^{5}$ who also reported no significant gender difference in pain or anxiety measure following tooth extraction. The last two authors however mentioned a persistently lower male score than females on several scales which is in keeping with the findings of this present study, in which mean pain score in females was persistently higher than that of males, though not statistically significant. Contrary to the finding that there is no significant difference in the mean pain score according to gender, Seymour et $\mathrm{al}^{7}$ reported a significant gender difference in the scores of present pain intensity, number of words chosen and total McGill pain rating in a study focused on pulpitis and pericoronitis. Dao and LeResche ${ }^{14}$ attributed the higher severity, frequency and duration of pain in women to sex differences in generic pain mechanisms and gender differences in social rules for pain expression.

The peak age incidence of presentation for odontogenic pain in this study was the third decade in line with findings of Macfarlene et $\mathrm{al}^{15}$ who observed a higher female incidence and peak age incidence of orofacial pain between 18 and 25 years.

In a study on orofacial pain carried out by MacMillan ${ }^{4}$, toothache and tooth associated sensitivity were the most common types of orofacial pain accounting for $27 \%$ and $15 \%$ of cases respectively. Also a Swedish study by Wolf et $\mathrm{al}^{16}$ reported a toothache prevalence of $56 \%$ among patients with orofacial pain while Seymour et $\mathrm{al}^{7}$ reported pulpitis and pericoronitis as the commonest form of dental condition that cause patient to seek treatment, all these studies emphasize the prominent role of caries related lesions as primary cause of orofacial pain.

Caries is a disease of urbanization with higher prevalence among the educated mainly due to influence of refined carbohydrate diet on caries development ${ }^{17,18,19,20}$, this was observed in the present study which took place in an urban area, a state capital and at the foremost tertiary health facility in West Africa. Akpata ${ }^{21}$ in a Nigerian study had earlier observed a higher prevalence of dental caries in urban dwellers when compared with rural dwellers and also observed that the scanty dental facilities in the country were located in the urban areas such as the present study centre.

Amongst patients with odontogenic lesions, pulpitis, apical periodontitis and its related complication of submandibular cellulitis presented the most severe pain according to VAS and verbal description of pain severity and also presented very high scores according to MPQ assessment which was only surpassed in severity by pain of tooth fracture (complicated crown fracture) which may also be considered as the traumatic variants of pulpitis and apical periodontitis. Dental caries and periodontal disease have previously been identified as the most common afflictions of the mouth ( kureishi 1998) ${ }^{22}$.

Among the periodontally involved lesion, the present study observed that two cases of impacted supplemental teeth presented the most severe pain this is in keeping with finding of Van Buren and Kleinknecht ${ }^{5}$ who also reported the highest pain severity scores in relation to impacted third molar.

The least pain severity was seen in patients with either tooth surface loss or failed restorations; Dentinal sensitivity usually follow tooth surface loss that may result from chronic attrition, abrasion or erosion, also dentinal sensitivity has been recognised as a result of partial dentinal fracture which may be a complication of failed amalgam filling. The resulting dentinal sensitivity usually present as persistent dull pain which is aggravated by extremes of temperature and exposure to air ${ }^{10,23}$.

Persistent dull pain after endodontic treatment despite complete radiographic healing was more frequent in patients with heavily restored teeth especially following prolonged delay in the restorations of the coronal $\operatorname{aspect}^{24}$. 
The fact that there was positive correlations between each pair of pain intensity measurement scales and especially between VAS and MPQ suggests that all the three scales could be used to assess pain severity of odontogenic lesions, furthermore the simultaneously use of two or more scales with consistent findings will further establish the pain severity. Positive correlation between the three measuring scales for chronic orofacial pain was previously reported by Russell Vickers ${ }^{10}$ and further confirmed by R.A Seymour et al ${ }^{7}$, who noted positive correlation between NWC, VAS and MPQ with particularly high correlation between PPI score of MPQ and VAS (r=0.69).

\section{Conclusion}

This study demonstrated a definite preponderance of pain of odontogenic origin over non odontogenic pain at our centre. It also showed that MPQ, VAS and NWC are useful pain rating scales for assessing the severity of orofacial pain generally. The scales showed significant discriminating power between pains of various aetiological categories, these are; caries related lesions, traumatized teeth, tooth surface loss, periodontal lesions and faulty restorations.

However, when the mean score of pain intensity is close as in the case of pulpitis and acute apical periodontitis, the three scales became ineffective in distinguishing the specific diagnosis, therefore these scales should only be used as adjunct to diagnosis as they may not be discriminatory enough for specific diagnosis.

\section{References}

[1]. Traebert J, de Lacerda JT, Fischer TK, Jinbo Y. Dental caries and orofacial pain trends in 12-year-old school children between 1997 and 2003. Oral Health Prev Dent 3, 2005, 243-8.

[2]. Omitola OG, Arigbede AO. Prevalence and Pattern of Pain Presentation among Patients Attending a Tertiary Dental Center in a Southern Region of Nigeria. J Dent Res Dent Clin Dent Prospect 4(2), 2010, 42-46.

[3]. Lou Y, MacMillan AS, Wong MC, Zheng J, Lam Cl. Orofacial pain condition and its impact on the quality of life in community dwelling elderly people in Hong Kong. J Orofac Pain 21, 2007, 63-71.

[4]. MacMillan AS, Wong MC, Zheng J, Lam MC. Prevalence of orofacial Pain and treatment seeking in Hong Kong Chinesse. $J$ Orofac Pain 20 (3), 2006, 218-25.

[5]. Van Buren J, Kleineknecht RA. Evaluation of the MPQ for use in Dental pain assessment. Pain 6, 1979, $23-33$.

[6]. Melzeck R. The McGill Pain Questionnaire: major properties and scoring methods. Pain 1, 1975, $277-99$.

[7]. Seymour RA, Simpson JM, Charlton JE, Phillips ME. An evaluation of length and end phrase of visual analogue scales in dental pain. Pain 21, 1985,177-85.

[8]. Fagade OO, Oginni FO, Adekoya-Sofowora CA. Age, Gender And Dental Perception. Internet Journal of Dental Science.

[9]. Vickers ER, Cousin MJ. Analysis of 50 patients with atypical odontalgia. Oral Surg Oral Med Oral Pathol Oral Radiol Endod 85, 1998, 24-32.

[10]. Vickers ER, Cousin MJ. Neuropathic orofacial pain part 1- prevalence and pathophysiology. Aus Endod, 26, 2000,19-26.

[11]. Shinal RM, Fillingim RB. Overview of orofacial pain: epidemiology and gender differences in orofacial pain. Dent Clinic North Am 51, 2007, 1-18.

[12]. Gerschman JA, Wright JL, Hall WD, Reade PC, Holwill BJ. Comparisons of psychological and social factors in patients with chronic orofacial pain and dental phobic disorders. Australian Dental Journal 32, 1987, 331-5.

[13]. Bush FM, Harkins SW, Harrington WG, Price DD. Analysis of gender effects on pain perception and symptom presentation in temporomandibular pain. Pain 53, 1993,73-80.

[14]. Dao TT, Leresche L. Gender differences in pain: J. Orofac. Pain 14, 2000, 169-84

[15]. Macfarlane TV, Blinkhorn AS, Davies RM, Kincey J, Worthington HV. Oro-facial pain in the community: prevalence and associated impact. Community Dentistry and Oral Epidemiology 30, 2002, 52-60.

[16]. Wolf. E, Petersson K, Petersson A, Nilner M. Long lasting orofacial pain: a study of 109 consecutive patients referred to a pain group. Swed Dent J 25, 2001, 129-136.

[17]. Ismail AI, Morison EC, Burt BA, Caffesse RG. National history of periodontal disease in adults, findings from Tecumseh periodontal disease study. Journal of Dental Research 69, 1990, 430-5.

[18]. Diehnelt DE, Kiyat HA. Socioeconomic factors that affect international caries level: Community Dent Oral Epidemiology 29, 2001,226-33.

[19]. Skudutyte R, Aleksejuniene J, Erickson HM. Dental caries in adult Lithuanians. Acta Odontol Scand 58, $2000,143-7$.

[20]. El-Nadeef MA, Adegbembo AO, Honkala E. The association of urbanization with the prevalence of dental caries among school children in Nigerian new capital territory. Int Dent J 48, 1998, 44-9.

[21]. Akpata ES. Oral health in Nigeria; Int Dent J 54, 2004, 361-6.

[22]. Kureishi A, Chow AW. The tender tooth, dentoalveolar, pericoronal and periodontal infections. Inf Dis Clin North Am 2, 1988,16382.

[23]. Brynjulfsen A, Fristad I, Grevstad T, Hals-Kvinnsland I. Incomplete fractured teeth associated with diffuse longstanding orofacial pain: diagnosis and treatment outcome. Int Endodon J 35, 2002, 461-66.

[24]. Polycarpou N, Ng YL, Canvas D, Moles DR, Gulabivala K. Prevalence of persistent pain after endodontic treatment and factors affecting it's occurrence in cases with complete radiographic healing. Int Endod J 38, 2002, 169-78. 
Pain scores of odontogenic orofacial lesions in a tertiary Hospital in sub Saharan West Africa.

Table I. Socio-demographic profile of respondents with odontogenic lesions

Variables

Age group (years)

11-20

21-30

$31-40$

41-50

51-60

$61-70$

$\geq 70$

TOTAL

Male to

Chi square comparison of age group distribution according to gender is not significant $\left.\mathrm{X}^{2}=2.89\right)$

\section{Marital status}

Single

68

54

$(\%)$

Married

0.0

$(0.0)$

Separated

0.0

$(0.0)$

Widow/widower

$\underline{0.0}$

122

$\underline{0.0}$

100

Chi square comparison of marital status according to gender is significant.

Females

Total

$\begin{array}{cll}\text { Frequency } & (\%) & \\ 21 & (13.6) & 42 \\ 55 & (35.5) & 99 \\ 27 & (17.4) & 54 \\ 19 & (12.3) & 31 \\ 14 & (9.0) & 22 \\ 12 & (7.7) & 16 \\ \underline{7} & \underline{(4.5)} & \underline{13} \\ 155 & 100 & 277\end{array}$

$(\mathrm{p}=0.822$ $\left(\mathrm{p}=.036 ; \mathrm{X}^{2}=10.29\right)$.

\section{Educational status}

$\begin{array}{lccccc}\text { No education } & 3 & (2.5) & 14 & (9.0) & 17 \\ \text { Primary } & 4 & (3.2) & 9 & (5.8) & 13 \\ \text { Secondary } & 26 & (21.3) & 36 & (23.2) & 62 \\ \text { Tertiary } & \underline{89} & \underline{(73.0)} & \underline{96} & \underline{(62.0)} & \underline{185} \\ \text { TOTAL } & 122 & 100 & 155 & 100 & 277\end{array}$

Chi square comparison of educational status according to gender is not significant. $\left(\mathrm{p}=0.093 ; \mathrm{X}^{2}=6.43\right)$.

Table II. Distribution and Comparison of Mean pain scores of the three pain measuring scales

Category of lesions

Caries Related

Tooth surface loss

Periodontal lesions

Traumatic dental injury

Faulty Restorations according to disease category.

\begin{tabular}{|c|c|c|}
\hline \multicolumn{3}{|c|}{ Mean Scores of Pain Severity } \\
\hline VAS & NWC & MPQ \\
\hline $6.0 \pm 2.1$ & $2.5 \pm 0.9$ & $28.7 \pm 12.1$ \\
\hline $3.3 \pm 1.2$ & $2.3 \pm 0.6$ & $17.8 \pm 7.0$ \\
\hline $5.7 \pm 2.3$ & $2.3 \pm 0.8$ & $23.3 \pm 11.3$ \\
\hline $5.6 \pm 2.0$ & $2.0 \pm 0.7$ & $30.0 \pm 13.4$ \\
\hline $3.4 \pm 1.7$ & $1.6 \pm 0.5$ & $23.2 \pm 9.5$ \\
\hline
\end{tabular}




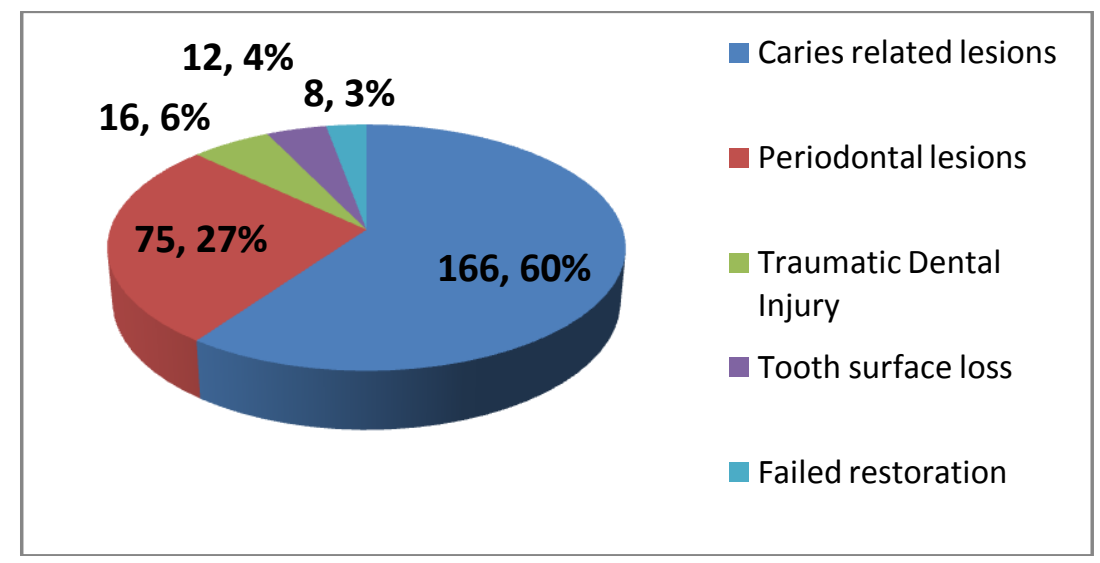

Figure 1: Lesion categories of orofacial pain of odontogenic origin.

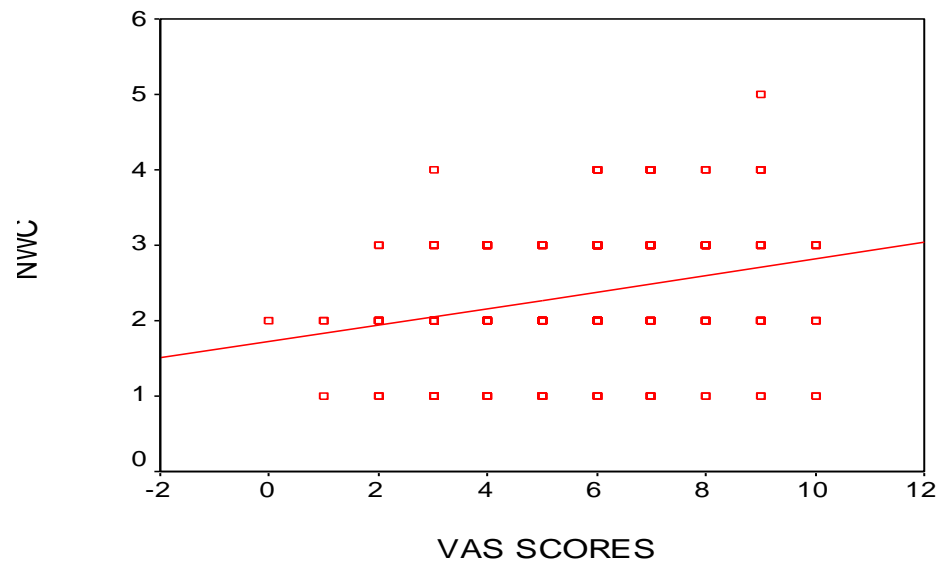

Figure 2: Correlations between the scores of VAS \& NWC among respondents $(\mathrm{r}=0.587 ; \mathrm{p}=\mathbf{0 . 0 0})$

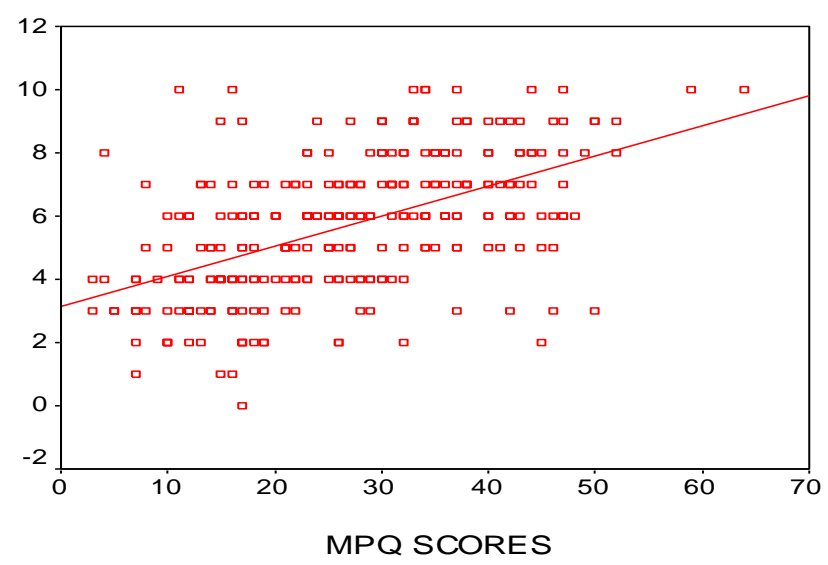

Figure 3: Correlations between scores of VAS \& MPQ among respondents $(\mathbf{r}=0.838 ; p=0.00)$ 


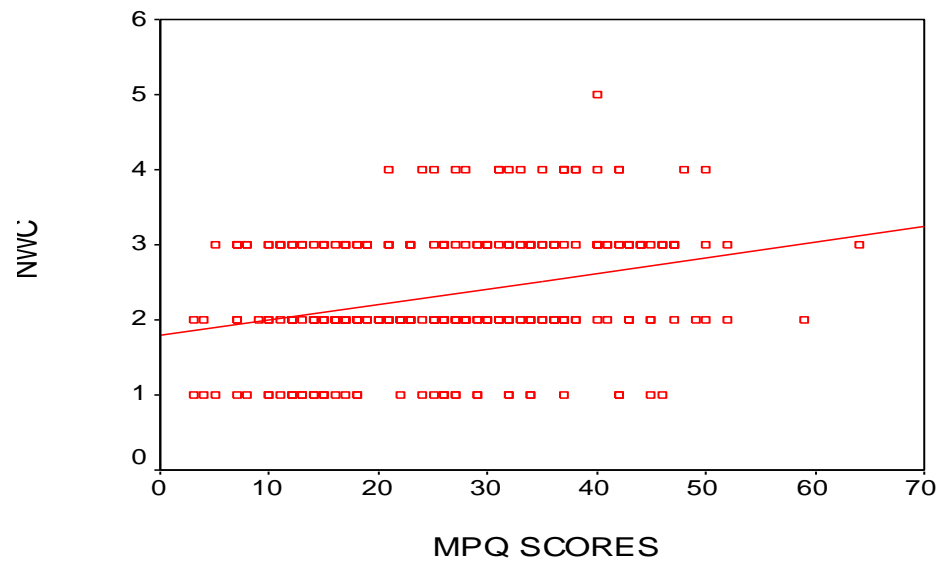

Figure 4: Correlation between scores of mpq \& nwc among respondents $(\mathrm{r}=0.599 ; \mathrm{p}=0.00)$.

Legend of figures:

Figure 1: Lesion categories of orofacial pain of odontogenic origin.

Figure 2: Correlations between the scores of VAS \& NWC among respondents

Figure 3: Correlations between scores of VAS \& MPQ among respondents.

Figure 4: Correlation between scores of mpq \& nwc among respondents. 